\title{
THE
}

$4-15-1987$

\section{Classical Spin Clusters: Integrability and Dynamical Properties}

\author{
N. Srivastava \\ Charles Kaufman \\ University of Rhode Island, ckaufman@uri.edu \\ Gerhard Müller \\ University of Rhode Island, gmuller@uri.edu \\ E. Magyari \\ R. Weber
}

See next page for additional authors

Follow this and additional works at: https://digitalcommons.uri.edu/phys_facpubs

Terms of Use

All rights reserved under copyright.

\section{Citation/Publisher Attribution}

Niraj Srivastava, Christopher Kaufman, Gerhard Müller, E. Magyari, R. Weber and H. Thomas. Classical spin clusters: integrability and dynamical properties. J. Appl. Phys. 61 (1987), 4438-4440.

Available at: http://dx.doi.org/10.1063/1.338402

This Article is brought to you for free and open access by the Physics at DigitalCommons@URI. It has been accepted for inclusion in Physics Faculty Publications by an authorized administrator of DigitalCommons@URI. For more information, please contact digitalcommons-group@uri.edu. 
Authors

N. Srivastava, Charles Kaufman, Gerhard Müller, E. Magyari, R. Weber, and H. Thomas

This article is available at DigitalCommons@URI: https://digitalcommons.uri.edu/phys_facpubs/120 


\title{
Classical spin clusters: Integrability and dynamical properties
}

\author{
N. Srivastava, C. Kaufman, and G. Müller \\ Department of Physics, University of Rhode Island, Kingston, Rhode Island 02881-0817 \\ E. Magyari, P. Weber, and H. Thomas \\ Institut für Physik, Universität Basel, CH-4056 Basel, Switzeriand
}

\begin{abstract}
A pair of exchange-coupled classical spins with biaxial exchange and single-site anisotropy represents a Hamiltonian system with two degrees of freedom for which the integrability question is nontrivial. We have found that such a system is completely integrable if the model parameters satisfy a certain condition. For the integrable cases, the second integral of the motion (in addition to the Hamitonian), which guarantees integrability, is determined explicitly. It can be reconstructed numerically by means of time averages of dynamical variables over all trajectories. In the nonintegrable cases, the existence of the time averages is still guaranteed, but they no longer define an analytic invariant, and their determination is subject to long-time anomalies. Our numerical calculation of time averages for two lines of initial conditions reveals a number of interesting features of such nonanalytic invariants.
\end{abstract}

Notwithstanding the overwhelming success of Maxwell's theory of classical electromagnetism for numerous applications, magnetism as a cooperative phenomenon in matter remained a mystery until the advent of quantum mechanics. The breakthrough came with the discovery by Heisenberg and Dirac of the exchange interaction as a purely quantum mechanical mechanism responsible for cooperative magnetism. Ironically the most immediate progress in understanding the physical properties of macroscopic systems of exchange-coupled electron spins was achieved in the context of theories in which these intrinsically nonclassical objects were adapted to the formalism of classical Hamiltonian dynamics. Classical spin dynamics of magnetic insulators, which employs classical counterparts of quantum-mechanical exchange Hamiltonians, has become very useful both in statistical mechanics and in magnetic materials research. However, only very recently attention has been given to nonintegrability effects in spin dynamics. ${ }^{2-5}$ Hamiltonian chaos in classical spin dynamics exhibirs a number of peculiar features associated with the following properties which are atypical for classical dynamical systems: the spin Hamiltonian is not of the type "kinetic energy plus potential energy"; the energy is bounded by a finite interval; the phase space is a compact manifold. The objective of the work reported here is to study time averages in nonintegrable dynamical systems and thus prepare the ground for a detailed study of nonintegrability effects in time-dependent correlation functions of classical spin systems.

Consider a system of $N$ localized classical three-component spins $\mathbf{S}_{l}, l=1, \ldots, N$ specified by some interaction Hamiltonian $H\left(\mathbb{S}_{1}, \ldots, \mathbb{S}_{N}\right)$. Its time evolution is governed by the Hamilton equation of motion

$$
\frac{d \mathbb{S}_{l}}{d i}=\left\{H, \mathbb{S}_{l}\right\}, \quad l=1, \ldots, N .
$$

The Poisson brackets for classical spin variables (the symplectic structure for classical spin dynamics) are constructed via the condition that the resulting Hamilton equation of motion ( 1 ) is consistent with the Heisenberg equation of motion for quantum spin operators:

$$
\left\{S_{l}^{\alpha}, S_{l}^{\beta}\right\}=-\delta_{l l}, \sum_{\gamma} \epsilon^{\alpha \beta \gamma} S \gamma_{l}
$$

If the classical spins $\mathbf{S}_{l}$ are expressed in terms of spherical coordinates as $\mathbf{S}_{l}=S\left(\sin \vartheta_{l} \cos \phi_{l}, \sin \vartheta_{l} \sin \phi_{l}, \cos \vartheta_{l}\right)$ then a set of canonical variables is given by $p_{l}=S \cos \vartheta_{l}$, $q_{l}=\phi_{l}$. Thus a system of $N$ classical spins specified by an energy function $H\left(\mathbb{S}_{1}, \ldots, \mathbb{S}_{N}\right)$ represents an autonomous Hamilonian system of $N$ degrees of freedom. The system is completely integrable if there exist $N$ distinct integrals of the motion in involution ${ }^{6}$ :

$$
I_{k}\left(\mathbb{S}_{1}, \ldots, \mathbb{S}_{N}\right)=\text { const }, k=1, \ldots, N,
$$

with $\left\{I_{k}, I_{k^{\prime}}\right\}=0$ for $k, k^{\prime}=1, \ldots, N$. A completely integrable $N$-spin system is characterized by the property that all phase-space trajectories are regular, i.e., confined to $N$-dimensional tori and described by a discrete Fourier spectrum. The $N$-tori are, in fact, obtained by the intersection of the $N$ $(2 N-1)$-dimensional hypersurfaces $I_{k}=$ const. If fewer than $N$ independent integrals of the motion in involution exist, the foliation of the phase space by invariant tori is incomplete, thus leaving room for new types of trajectories in addition to regular ones: trajectories whose course through phase space is strikingly erratic and extremely sensitive to slight changes in initial conditions and whose Fourier spectrum is continuous. These are the chaotic trajectories?

In a previous study, ${ }^{4}$ we have studied the integrability problem for a system of two exchange-coupled spins, specified by a Hamiltonian of the general form

$$
H=\sum_{\alpha=x y z}\left\{-J_{\alpha} S_{1}^{\alpha} S_{2}^{\alpha}+(1 / 2) A_{\alpha}\left[\left(S_{1}^{\alpha}\right)^{2}+\left(S_{2}^{\alpha}\right)^{2}\right]\right\}
$$

which inciudes both exchange and single-site anisotropy. We have shown that an independent integral of the motion (in addition to $H$ ) quadratic in the spin variables exists if and only if the model constants satisfy the equation

$$
\begin{aligned}
& \left(A_{x}-A_{y}\right)\left(A_{y}-A_{z}\right)\left(A_{z}-A_{x}\right) \\
& \quad+\sum_{\alpha \beta \gamma} \sum_{\text {cycl }(x y z)} J_{\alpha}^{2}\left(A_{\beta}-A_{\gamma}\right)=0 .
\end{aligned}
$$


If $A_{x}=A_{y}=A_{z}$, the second invariant has the form

$$
\begin{aligned}
I= & -\sum_{\alpha \beta \gamma=\operatorname{cycl}(x y z)} J_{\alpha} J_{\beta} S_{1}^{\gamma} S_{2}^{\gamma} \\
& +\frac{1}{2} \sum_{\alpha=x y z} J_{\alpha}^{2}\left[\left(S_{1}^{\alpha}\right)^{2}+\left(S_{2}^{\alpha}\right)^{2}\right] .
\end{aligned}
$$

if not all three $A_{c x}$ are equal, it is given by

$$
I=\sum_{\alpha=x y z} g_{s x} S_{1}^{\alpha} S_{2}^{\alpha}
$$

where

$$
\begin{aligned}
g_{\alpha}= & J_{\alpha}\left(J_{\alpha}+J_{\beta}+J_{\gamma}\right)+\left(A_{\alpha}-A_{\beta}\right) J_{\gamma} \\
& +\left(A_{\alpha}-A_{\gamma}\right) J_{\beta}-\left(A_{\alpha}-A_{\beta}\right)\left(A_{\alpha z}-A_{\gamma}\right)
\end{aligned}
$$

with $\alpha \beta \gamma=\operatorname{cycl}(x y z)$. Moreover, we have provided numerical evidence that the violation of condition (5) implies not only the nonexistence of a quadratic invariant but the nonexistence of a second independent analytic invaxiant in generai.

For the present work we consider an $X Y$-type two-spin model with single-site anisotropy in the $x y$ plane

$$
\begin{aligned}
H_{\alpha}= & -\left(S_{1}^{x} S_{2}^{x}+S_{1}^{y} S_{2}^{y}\right)+(1 / 2) \alpha\left[\left(S_{1}^{x}\right)^{2}\right. \\
& \left.+\left(S_{2}^{x}\right)^{2}-\left(S_{2}^{y}\right)^{2}-\left(S_{2}^{y}\right)^{2}\right],
\end{aligned}
$$

which is nonintegrable except for $\alpha=0, \pm 1$. In Fig. 1 we show a number of phase-space trajectories for the case $\alpha=-1 / 2$ of this model. All trajectories shown are located on the same $3 D$ energy hypersurface $E=-0.09957501$ in $4 \mathrm{D}\left(\vartheta_{1}, \phi_{1}, \vartheta_{2}, \phi_{2}\right)$ space. For the purpose of better visualization, Fig. 1 actually shows only the projection onto the $\left(\vartheta_{1}\right.$, $\phi_{1}$ ) plane of the two Poincare surfaces of section of these trajectories which are defined by (a) $\hat{\vartheta}_{2}=\pi / 2, \dot{\vartheta}_{2}<0$ and (b) $\vartheta_{2}=\pi / 2, \dot{\vartheta}_{2}>0$. In this representation, regular trajectories appear as $1 \mathrm{D}$ objects (lines) if they are guasiperiodic

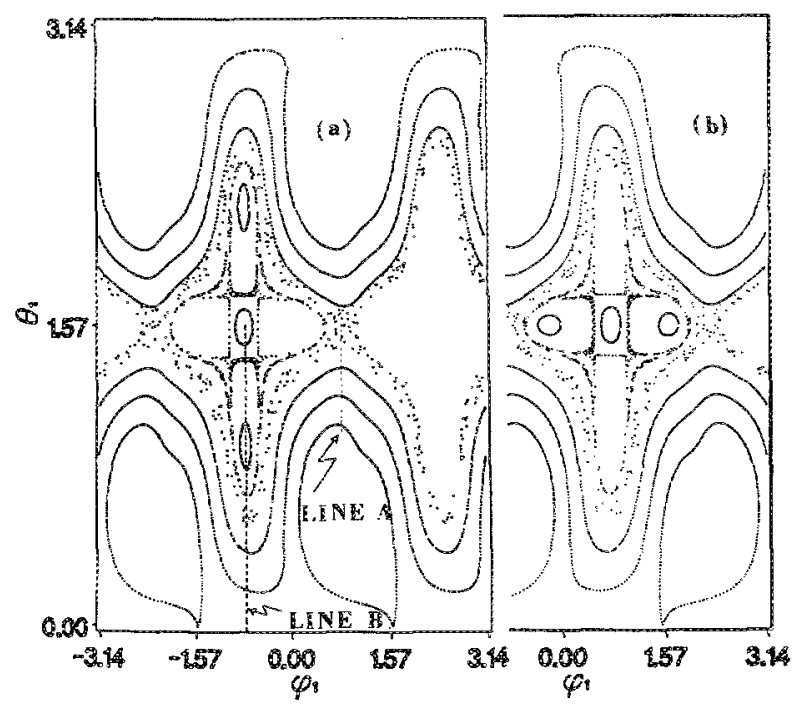

FIG. 1. Various phase-space trajectories of the nonintegrable classical twospin model $H_{c}$ with $\alpha=-1 / 2$, all for the same energy $E=-0.09957501$. Shown is the projection onto the $\left(\vartheta_{1}, \phi_{1}\right)$ plane of the two Poincare surfaces of section of these trajectories which are defined by (a) $\vartheta_{2}=\pi / 2, \dot{\vartheta}_{2}<0$ and (b) $\vartheta_{2}=\pi / 2, \dot{\vartheta}_{2}>0$. The dashed lines $A$ and $B$ specify two sets of initial conditions for which time averages of a dynamical variable over single trajectories are presented in Fig. 2. or as finite sets of points if they are periodic. The chaotic trajectories, on the other hand, are represented by more complicated sets of phase points, which spread over $2 \mathrm{D}$ regions on the $\left(\vartheta_{1}, \phi_{1}\right)$ plane.

The equations of motion (1) for our model (8) are invariant under six discrete symmetry transformations: the three twofold rotations about the coordinate axes $S^{\infty}, S^{y}, S^{z}$, in spin space, and the three refiections at the corresponding coordinate planes combined with time reversal. Whereas the Poincaré hyperplane $\vartheta_{2}=\pi / 2$ is invariant under al six transformations, the direction of any trajectory through this plane is left invariant only by two transformations: (i) $\left(S^{x}, S^{y}, S^{z}\right) \rightarrow\left(-S^{x},-S^{y}, S^{z}\right)$, which corresponds to $(\vartheta, \phi) \rightarrow(\vartheta, \phi \pm \pi)$, (ii) $\left(S^{x}, S^{y}, S^{z}\right) \rightarrow\left(S^{x}, S^{y},-S^{z}\right)$ combined with time reversal, which corresponds to $(\vartheta, \phi) \rightarrow(\pi$ $\vartheta, \phi)$. The Poincare cut of any trajectory then either has the symmetries (i) and (ii) or is transformed into the cut of a symmetry equivalent trajectory. In Fig. 1 we observe that one of the chaotic trajectories (the most prominent one) appears to have the full symmetry $(i+i i)$, whereas the regular trajectories break either symmetry (ii) (top and bottom) or symmetry (i) (center). That particular chaotic trajectory acts as the separatrix between two types of regular motion: precession of spin $S_{1}$ about the $z$ axis (top and bottom), accompanied by a considerable amount of nutation, and quasiperiodic oscillations of various complexity without precession (center). There are two pairs of primary fixed points on the axis $\vartheta_{1}=\pi / 2$ in Fig. 1: a pair of hyperbolic fixed points on the chaotic separatrix and a pair of elliptic fixed points in between. Furthermore, we can identify a pair of seconalary elliptic fixed points representing periodic trajectories of period 2 (in the cut), which originate from destroyed rational tori about the primary fixed point. These secondary elliptic fixed points are accompanied by an equal number of hyperbolic ones and surrounded by chaotic separatrices. Similar patterns occur at the rational tori of secondary elliptic fixed points and so on, ad infinitum.?

Even though the model specined by Hamiltonian $H_{c z}$ becomes nonintegrable as the parameter $\alpha$ is assigned values different from $0, \pm 1$, which implies the nonexistence of a second analytic invariant in addition to $H_{\alpha}$, the abundance of invariant tori observed in the phase flow suggests that fragments of the second invariant $I$ survive in some form. In the following we outline a procedure for the numerical reconstruction of these fragnents.

Let us first consider the completely integrable cases $\alpha=0, \pm 1$, for which the two analytic invariants necessary for complete integrability are $H_{\alpha}$ itself and $I$ as given in Eq. (7) by analy tic construction. If the second analytic invariant $I$ were not known explicilly, it could be reconstructed numerically as follows: Pick any dynamical variable $X$ which is independent of $H_{\alpha}$. The time average of $X$ over any (regular) trajectory is then, by construction, an invariant. $T t$ is, in fact, an analytic function of the initial conditions and can be identified as the second independent integral of the motion

$$
\langle X\rangle \equiv \lim _{T \rightarrow \infty} \frac{1}{T} \int_{0}^{T} d t X(t)=I\left(\mathbf{S}_{1}, \mathbf{S}_{2}\right) .
$$

In nonintegrable cases such as $\alpha=-1 / 2$, the Birkhoff theorem ${ }^{8,9}$ guarantees that the time average Eq. (9) still ex- 

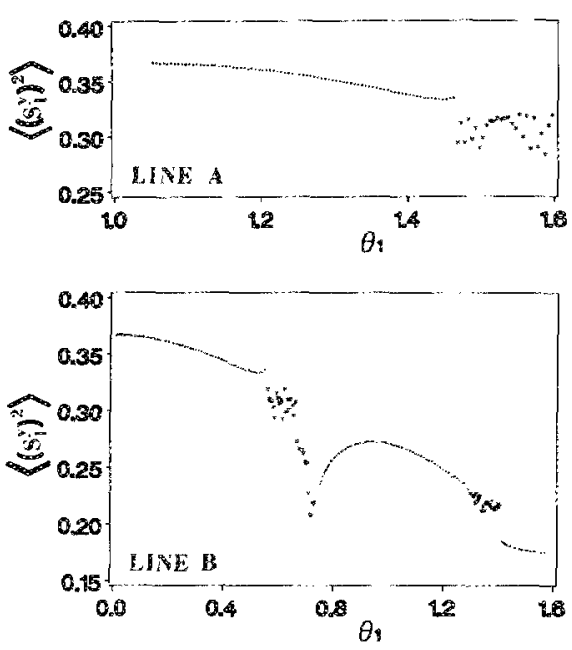

FIG. 2. Time average $\left\langle\left(S_{i}\right)^{2}\right\rangle$ over single (regular or chaotic) trajectories as a function of $\vartheta_{1}$ for initial conditions along lines $A$ and $B$ shown dashed in Fig. 1. The dots represent time averages which have converged to within 1 part in $10^{4}$. The time averages over some chaotic trajectories (represented by asterisks) are subject to long-time anomalies. The estimated numerical uncertainties are up to 1 part in 30 for those points.

ists for almost all trajectories, even chaotic ones. However, the quantity $I\left(\mathbf{S}_{1}, \mathbf{S}_{2}\right)$ is no longer an analytic function; its nonanalyticities are, of course, associated with the presence of chaotic trajectories in phase space. We may call it a nonanalytic invariant.

With the calculation of time averages (9), we can thus investigate the most interesting question of the persistence and smoothness of residues of the second integral of the motion in the nonintegrable case in regions of the energy hypersurface depicted in Fig. 1, where invariant tori are predominant, and in regions where chaotic trajectories are predominant. For this purpose we consider two lines $A$ and $B$ on the energy hypersurface $E=-0.09957501$ intersected by the Poincare hyperplane $\vartheta_{2}=\pi / 2$. The projections of these two lines onto the $\left(\vartheta_{1}, \phi_{1}\right)$ plane are shown dashed in Fig. 1. In Fig. 2 we have plotted the time average $\left\langle\left(S_{1}^{y}\right)^{2}\right\rangle$ as a function of $\vartheta_{1}$ for initial conditions along lines $\mathrm{A}$ and $\mathrm{B}$.

Line A starts out in a predominantly regular region, where chaotic trajectories are very restricted in their course and range and then enters the broad chaotic separatrix region. This has its reflection in Fig. 2 as follows: The nonanalytic invariant $\left\langle\left(S_{1}^{y}\right)^{2}\right\rangle$ appears to be fairly smooth in the regular region, but its nonanalytic nature is dramatically apparent at the border to and inside the chaotic region. However, we must bear in mind that within the regular region there is (hike everywhere in phase space) a dense population of chaotic trajectories and that within the chaotic region there is an abundance of small islands of regular trajectories, all of which affect the smoothness of the invariant $\left\langle\left(S_{1}^{y}\right)^{2}\right\rangle$ on ever smaller scales. The effect of larger scale regular is- lands on the quantity $\left\langle\left(S_{\frac{1}{y}}^{y}\right)^{2}\right\rangle$ can be investigated along line $\mathrm{B}$ of Fig. 1. It shows three relatively smooth pieces corresponding to the regular region outside the chaotic separatrix and the two regular islands around a secondary and a primary elliptic fixed point. It is interesting to note that the extrapolated curve of one regular region does not seem to connect to the extrapolated curve of a neighboring regular region across a narrow band of chaos. Also, the irregular pattern of points in the chaotic intervals of Fig. 2 suggests that the phase fow might not be ergodic within those regions; ergodic flow would imply that $\langle X\rangle$ is the same for all initial conditions in a given connected region.

At this point we should add a word on the practical aspects of the calculation of time averages. Even though the Birkhoff theorem guarantees the existence of time averages for virtually all trajectories, in practice one may sometimes encounter difficulties because of long-time anomalies. We have identified two mechanisms which seem to be responsible for such anomalies: (i) The chaotic motion may appear to be superimposed on a periodic component with widely separated phase slips. (ii) Chaotic trajectories may stay for long times in regions bounded by "cantori," i.e., by nearly intact tori which have decayed to Cantor sets. This mechanism has its analogy in the critical slowing down in the theory of phase transitions.

Evidently all these results along with our qualitative interpretation raise a number of interesting questions which in turn call for a more extensive investigation of time averages and nonanalytic invariants in nonintegrable dynamical systems. A detailed study is underway. Moreover, a satisfactory understanding of these questions appears to be a prerequisite for a successful analysis of time-dependent correlation functions, which is the actual goal of our endeavor. The work in Basel was supported by the Swiss National Science Foundation and the work at URI by the Council on Research of the University of Rhode Island, by a grant from Research Corporation, and by the U. S. National Science Foundation, Grant Number DMR-86-03036.

M. Feingoid and A. Feres, Physica 9D, 433 (1983); M. Feingold, N. Moiseyev and A. Peres, Phys. Rev. A 30, 509 (1984).

${ }^{2}$ K. Nakamura, Y. Nakahara and A. R. Bishop, Phys. Rev. Lett. 54, 861 (1985); K. Nakamura and A. R. Bishop, Phys. Rev. B 33, 1963 (1986). ${ }^{3}$ H. Frahm and H. J. Mikeska, Z. Phys. B 60, 117 (1985).

${ }^{4}$ E. Magyari, H. Thomas, R. Weber, C. Kaufman, and G. Müller, Z. Phys. B $65,363(1987)$.

G. Müller, Phys. Rev. A 34, 3345 (1986).

sV. I. Amold, Mathematical Methods of Classical Mechanics (Springer, New York, 1978), p. 271.

${ }^{7}$ A. J. Lichtenberg and M. A. Lieberman, Regular and Srochastic Motion (Springer, New York, 1983).

${ }^{8}$ A. I. Khinchin, Mathematical Foundations of Statistical Mechanics (Dover, New York, 1949), p. 19.

${ }^{9}$ R. Abraham and J. E. Marsden, Foundation of Mechanics, 2nd ed. (Benja$\mathrm{min} /$ Cummings, Reading, 1985), p. 238. 